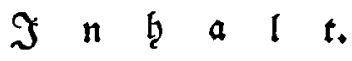

I. Heber bie $\mathfrak{R}$ upfer.

1) Sifforifte Supfer. $=s=$ E. XI

2) Sanofdaftlide Supfer. $\quad=$ XIII

$$
\text { II. } \mathfrak{E} \mathfrak{u} \mathfrak{f} \mathfrak{f p i e \mathfrak { l }} \text {. }
$$

Die Sdiffahrt. In eittem afte. Yoon

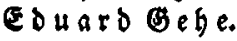
165

\title{
III. Ergáglungen.
}

Die weike Rofe. Pon E. 2 . Conteffa. 14 Die Yungfrau vout See. Bon $\mathfrak{R}$ ind. $\quad 67$

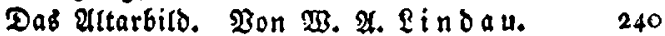
Die orei Góbne. Mon \&. Brathann. 316

IV. Geoidte.

Banlenfee (Graf) Jüngling auf dem See. $=292$ Sdimer. Mit Melodie von C. M. von $\mathfrak{B} \in \mathfrak{b} e$ r. 
IV

Bradmann (quife) Dab feuer. = Soffnung. = = $=365$ Eaftelli (I. F.) Die \$nonftranje. $\quad 206$

Der unglitctitie rag. $\quad=310$

Fórfer (Garl) lied uno Riebe. =

Atukenbleiben Der Geliebten. = $\quad 3^{67}$

$\mathfrak{H a}$ a $\mathfrak{g}(\mathbb{F}$.) $\mathfrak{A n}$ Melmar. $\quad=164$

fidi'b Ergablung. = $=298$

Mein Bang. $\quad=\quad=313$

Der Menith und Dic Blume. $=36+$

Der erfte Súk. = = $\quad=306$

Efolic. = = $=374$

Szell (Theodor) Brillenjágetlico. = $=381$

Şobengaufen (Elifa von) Tag uno

Radt. $=300$

Nalfreutb (Frietrid) (Br.v.) Warnung. $\quad 58$

Die brei Éfuane. $==231$

find (F.) Die Wallfahrt. = = I

Das Ritht im Balbe. Mit Melodie von E. M. 'v. 'Beber. = 238

Das Madben am badue.., Rit Mer. lobie von 2 . Met feffel. 307

Marieden am Sarge. Mit Melo= bie von $\mathfrak{A}$. C. $\mathfrak{F}$ ú $\mathrm{r}$ ftena 
Det dreifathe Hímmet. Mit Melo= bie von Dokauer. $=$ S.370

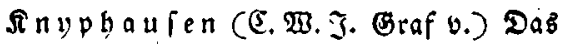

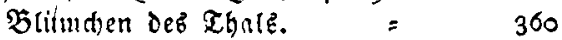
situhn (F.) Willelum $\mathfrak{P e n n . ~} \quad=\quad 223$ Inl leben. = $=281$ Iangbein (R. F. (5.) Die Reife Des 3itutiet Breitupfes. = 143

Soeben (D. S. (5r. von) Wonne bes Ditterb. Fibeinfahrt. $=$ - 372

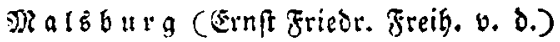
Sibulla Langmartel. = Reuffer. Preis ber Mufen. $\quad=\quad 287$ Rioba (Friebr. Sirug v.) 2 n bie 26 end $=$ róthe. = $=$ Der Miffionair. 59 Der Miffionair. $=\quad=128$ Die goldene 2lue. $=101$

Rorbfern (Arthur vom) Sditer. $\quad 54$ Seitit. = = 55

Buftav Abolfs Şalaftagen. = $\quad 135$

$\mathfrak{R a l l}$ (Witbetunine) Abnung in $\mathfrak{A}$ ugar: ten. $=297$ Refe. Pfyde's Entferferung. = jor 
VI

Sゅ il

lobie von $\mathcal{A}$. Nethfeffel. S. 306

Streffus (Nar) Diefegende vom Bis fdofe su Rerfeburg. = 150

Diebge (ङ. 2A.) Rebervohl. = 49

Die Stimne in Walbe. = 6r

Pautinentag. = = 296

Trinflied. $=376$

Wilibalo $M(e x i b$, Walpurgisnad)t. $\quad$ I62

Die farben. = $\quad 204$

V. Egataden und $\Re$ atb fed

von Eaftelli, Senriette von Sogen=

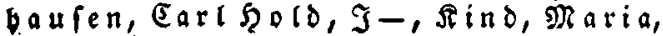

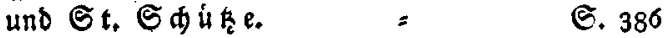

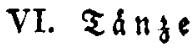

mit bagu gegóriger Mufie, von $\mathfrak{R}$ a 4 er y, $\Omega \dot{c}=$ niglid, Preú, Ballettmeifter uno Solotánger in Bertin.

VIT. Mifil.

Die bem diekjánrigen Jahrgange beigefingten Melodicen finb son (Ear Mariabon Beber 
(zu 5. 238. und 359.) R. IRethfeffet (zu

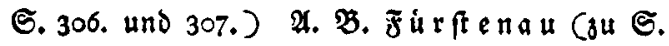
362.) und Dokg a er (zu S.37०.)

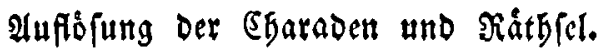

Dic Zuffofung Der im vorjågrigen Tafdens budse befindliden Efataven und $\mathfrak{R}$ ithfel if:

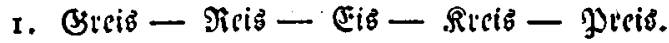
2. Nope und Tiros. 3. Dev Butel, als Sn: frument und als Figut. 4. Buthbandel. 5. Nadhs Druck. 6. Stalien und Eapitalien. 7. Tlugenliso.

\section{Dructefiet.}

Seite 217. Beile t. lies: Sidngelein

- 222. - 9 . lies : euth ff. aut

- 273. - 22. liez: nie fi. eine 